Method The pharmacist would attend the consultant-led morning handover or would liaise with the nurse in charge on the ward to establish discharges and transfers for that day or over the weekend if on a Friday. The most urgent discharges and any complex patients were prioritised. The EPR system would be used to generate the EDL's, transcribe the medicines for discharge and add any other relevant written information. Any medication related issues would be clarified with the medical team. The prescription would be handed over to the medical team to be reviewed and signed. This would then be dispensed and checked by the pharmacy team. The patient/parent or carer would be counselled on their medications. Data was collected from November 2018 - March 2019, this included time informed about discharge, time EDL started, time EDL printed and time EDL completed. Other data collected included if any additional written information was provided to the GP and if any amendments were required after the doctor had reviewed the prescription. The data was inputted into an Excel spreadsheet and was compared against August - October 2018.

Results 152 discharge prescriptions were included in the service. The data was compared to the data from August - October 2018 which showed more than double of the prescriptions were completed in the morning between 9am12 noon (compared to 12 noon-5.30pm) since the service started. Less prescription needed amendments at the point of screening and more prescriptions included additional medication related information. The quality of the prescriptions had improved and completing prescriptions earlier meant timely discharges, improved bed utilisation and improved patient quality. Positive feedback was given by patients, doctors and nurses as well as the rest of the ward teams.

Conclusion Communication has improved between the hospital and community care, as well as patient satisfaction and bed availability. A future development would be to introduce prescribing pharmacists within medical teams to streamline the discharge prescription process further, freeing up medical time and increasing the focus on medicines optimisation for all patients.

\section{P27 IMPACT OF HAVING A PAEDIATRIC MEDICINES MANAGEMENT PHARMACY TECHNICIAN IN A DISTRICT GENERAL HOSPITAL}

Rebecca Le Maistre*. Northampton General Hospital

10.1136/archdischild-2020-NPPG.36

Aim Patients are more likely to experience a 'medicines-related safety incident' when medicines reconciliation happens more than 24 hours after admission to an acute setting, ${ }^{1}$ according to the National Institute for Health and Care Excellence (NICE). The study aimed to assess the impact on medicine reconciliations following the introduction of a dedicated Paediatric Medicines Management Pharmacy Technician to the paediatric wards at a District General Hospital (DGH).

Methods Data has been routinely collected by the pharmacy department over of a number of years showing the time of medicines reconciliations compared with the time of hospital admission. This data shows the number of medicine reconciliations that were completed within 24 hours of hospital admission and the number that were not completed within 24 hours. The data is routinely collected on the Thursday of the first full week of every month. All patients that were admitted to the paediatric wards were included in this data. The service is only funded Monday to Friday through the Child Health Department of the DGH. This data excludes neonates admitted to the Neonatal Intensive Care Unit. Data was collected from 83 paediatric patients in March/April/May 2017 and 78 paediatric patients in March/April/May 2019.

Results Data collected for the paediatric patients over March/ April/May 2017 showed that around 21.7\% of all paediatric patients admitted to the wards had a completed medicines reconciliation within 24 hours. The data collected over the same period in 2019 showed that $85 \%$ paediatric patients admitted to the wards had a completed medicines reconciliation within 24 hours.

Conclusion This study was useful in demonstrating the effectiveness of introducing a dedicated Paediatric Medicine Management Pharmacy Technician to the paediatric wards in a DGH. It showed that the proportion of medicine reconciliations within 24 hours prior to the change was very low, but after the change it was very high with nearly all patients having a completed medicines reconciliation within 24 hours. Prior to the introduction of a dedicated Paediatric Medicines Management Pharmacy Technician, the paediatric wards at this DGH were not meeting the standards set by NICE regarding medicines reconciliations within 24 hours of being admitted to an acute setting. After the introduction the paediatric wards were meeting these standards. By meeting NICE guideline QS120 Medicines Optimisation, the DGH has reduced the likelihood of medicines-related safety incidents. With the introduction of a dedicated Paediatric Medicines Management Pharmacy Technician there have been many other benefits. These include counselling to parents/children on the use of their medicines; checking of patients' own medicines to see if they are still fit for purpose; advice to parents about unlicensed medicines and why they are used; where to obtain further supplies when new medicines have been started; and assisting parents and GP surgeries with any supply issues.

\section{REFERENCE}

1. National Institute for Health and Care Excellence. Medicines Optimisation (internet). (London):NICE; March 2016.(Quality Standard [QS120]). Available from www.nice.org.uk/guidance/qs120//chapter/Quality-statement-4-Medicines-reconciliation-in-acute-settings

\section{P28 GENTAMICIN-RELATED INCIDENTS IN NEONATES BEFORE AND AFTER THE INTRODUCTION OF ELECTRONIC PRESCRIBING AND MEDICINES ADMINISTRATION (EPMA)}

Kimberly Mak*. Leeds Teaching Hospitals NHS Trust

\subsection{6/archdischild-2020-NPPG.37}

Aim Gentamicin is widely used to treat early neonatal sepsis as part of a regimen recommended by NICE. ${ }^{1}$ However, it is frequently implicated in clinical incidents relating to errors in prescribing and administration. This project aimed to evaluate whether the introduction of ePMA had an effect on the frequency and type of incidents that occur relating to the use of gentamicin in neonates.

Method A paper gentamicin prescription chart was used from July 2013 until the implementation of ePMA on 28th January 2019. Using ePMA, prescribers were encouraged to use a pre-set template for 'neonatal early onset sepsis', 